\title{
Mineração de dados aplicada a educação: um estudo comparativo acerca das características que influenciam a evasão escolar
}

\author{
Kennet E. A. Calixto ${ }^{1}$, Caetano V. N. Segundo ${ }^{1}$, Renê P. de Gusmão ${ }^{2}$ \\ ${ }^{1}$ Departamento de Sistemas de Informação - Faculdade Paraíso do Ceará (FAP-CE) \\ Juazeiro do Norte - CE - Brazil \\ ${ }^{2}$ Departamento de Computação - Universidade Federal de Sergipe (UFS) \\ São Cristovão - SE - Brazil \\ \{kennet.emerson, caetanov120\}@gmail.com, rene@dcomp.ufs.br
}

\begin{abstract}
This article aimed to construct an analytical study to identify the variables related to school dropout, by analyzing data from the official National School Census in the years 2014, 2015 and 2016 from the states of Ceara and Sergipe. The CRISP-DM methodology was used to understand, prepare and model the dataset. The SPSS tool was used in the data separation process. The analysis were made by using the SPSS and RapidMiner tools that were used to apply the Rule Induction and Logistic Regression techniques. The generated models had an accuracy of around 87\%. In additional, variables as age, educational stage, teaching modality, existence of laboratories in the school, and its location stood out as influential variables in school dropout.
\end{abstract}

Resumo. Este trabalho teve como objetivo a construção de um estudo para identificar as variáveis concernentes à evasão escolar, utilizando os dados do censo educacional de 2014, 2015 e 2016 dos estados de Ceará e Sergipe. A metodologia CRISP-DM foi usada para entender, preparar e modelar os dados. Na separação dos dados foi utilizado a ferramenta SPSS. As análises se deram por meio do SPSS e do RapidMiner, utilizadas para empregar técnicas de Indução de Regras e Regressão Logística. Os modelos criados apresentaram acurácia em torno de $87 \%$. Além disso, a idade, etapa de ensino, modalidade de ensino, existência de laboratórios e localização da escola se destacaram como variáveis influentes na evasão escolar.

\section{Introdução}

Nos últimos anos, a educação passou por muitas mudanças que se configuraram como melhorias estruturais para as instituições educacionais. Embora tais mudanças tenham possibilitado a escolarização de milhões de estudantes da educação basica, dados recentes mostram que o Brasil ainda está entre os dez países com o pior desempenho nas áreas de ciência, matemática e leitura [OECD 2015]. Além de apresentar problemas relacionados ao desempenho educacional, o país também enfrenta desafios como o analfabetismo ainda não erradicado, o abandono e a evasão escolar. Este último item citado destaca-se entre os demais, pois ele pode posteriormente evoluir para o abandono, o analfabetismo e também a distorção idade/série (por exemplo, em casos que o indivíduo retorna a vida acadêmica para conclusão de sua formação após algum tempo fora da escola). 
A evasão escolar ocorre por diversas causas, que estão diretamente relacionadas a qualidade da educação oferecida pela instituição de ensino frequentada pelo aluno, o ambiente escolar, a relação familiar dele, o meio social em que ele vive (onde deve-se levar em consideração fatores como a qualidade de vida) e motivos concernentes à vida pessoal do próprio aluno. Em [Brasil 2006] são claramente apresentados os motivos da evasão e do abandono diretamente relacionados a problemas na vida social do aluno, que evade por motivos como a gravidez na adolescência, a necessidade de trabalhar para auxiliar na renda familiar ou a idade avançada decorrente de reprovações que acabam instigando o aluno a se sentir deslocado.

Tais eventos acabam demandando ações governamentais com o objetivo de superar o quadro problemático gerado pela evasão, o abandono, o analfabetismo e os demais problemas educacionais da contemporaneidade. O Programa Brasil Alfabetizado é um exemplo de programa que tem como meta a redução da desigualdade educacional e é voltado para a alfabetização de jovens, adultos e idosos [Diniz et al. 2014].

É importante ressaltar que o abandono e a evasão são dois conceitos diferentes. Embora estes conceitos estejam relacionados, o abandono se caracteriza quando o aluno deixa de frequentar as aulas e compromete todo o ano de estudo, mas retorna no ano seguinte. A evasão, por sua vez, ocorre quando o aluno abandona os estudos, mas não retorna a escola no ano seguinte para dar continuidade aos estudos [BRASIL 2012].

As pesquisas feitas no contexto da evasão escolar no Brasil são construídas levando em consideração análises isoladas de conjuntos de dados, ou seja, não são feitas comparações com conjunto de dados de origens distintas [Bezerra et al. 2016], que se realizadas, poderiam fornecer bases mais sólidas para inferir os fatores da evasão escolar. Outro ponto de igual importância é a abordagem fazendo uso de dados de um determinado contexto histórico, ou seja, uma análise que considera dados de um conjunto de anos subsequentes, visto que a educação sofre constantes mudanças influenciadas pelos avanços econômicos, políticos, sociais e tecnológicos.

Nesse contexto, este trabalho teve como objetivo a construção de um estudo analítico a fim de identificar as variáveis concernentes à evasão escolar e comparar a relação entre os estados analisados entre os anos de 2014,2015 e 2016 dos estados de Ceará e Sergipe. Para isso será necessário analisar todos os dados relacionados aos alunos, escolas e docentes; aplicar métodos de mineração de dados e comparar os resultados entre os anos e os estados.

A estrutura desta pesquisa está organizada da seguinte forma. Na seção 2 é apresentada uma revisão sobre os trabalhos relacionados ao tema desta produção. A seção 3 apresenta as técnicas aplicadas, que vão desde o processo adotado e a descrição da base, até o pré-processamento e as técnicas de mineração aplicadas no conjunto de dados analisados. Os resultados das análises construídas sobre os dados são discutidos na seção 4. As considerações finais e os trabalhos futuros são apresentadas na seção 5.

\section{Trabalhos Relacionados}

[Digiampietri et al. 2016] apresenta um estudo acerca da evasão escolar do curso de graduação em Sistemas de Informação da Universidade de São Paulo (USP). A pesquisa teve o objetivo de classificar os discentes com risco de desistência, em que o abandono é 
VI Congresso Brasileiro de Informática na Educação (CBIE 2017)

Anais do XXVIII Simpósio Brasileiro de Informática na Educação (SBIE 2017)

caracterizado pelo bacharel que não conclui o curso. De acordo com o trabalho, a base de dados considerou apenas variáveis relacionadas às notas obtidas em toda a graduação, desprezando variáveis como características dos docentes, estrutura da universidade e variáveis pessoais relacionadas aos discentes. A classificação é realizada pelo algoritmo Rotation Forest.

Em [Agaoglu 2016], as técnicas de mineração de dados são utilizadas com foco não no discente, mas sim no docente. A pesquisa objetiva mensurar a qualidade e desempenho docente, utilizando dados coletados de forma aleatória dos alunos. O estudo utilizou exclusivamente um questionário com base nas opiniões de cada aluno, de forma que não levasse em consideração a qualidade do local trabalho do docente e nem o seu grau de especialização relacionado ao seu desempenho de ensino.

Os autores em [?], apresentam uma pesquisa realizada nas escolas públicas no estado do Pernambuco com o objetivo de identificar um perfil de aluno com predisposição a evasão. Através de técnicas de mineração de dados foram aplicados algoritmos de Árvore de Decisão, Indução de Regra e Regressão Logística nos dados. O estudo, no entanto, não contemplou comparações com outros estados e considerou apenas a evasão de 2011 para 2012.

Em [Nunes 2015] é apresentada a aplicação de mineração de dados em ambientes virtuais com o propósito de permitir mudanças no planejamento pedagógico. Os experimentos são realizados no ambiente OpemSim, onde através de um laboratório virtual de química as variáveis relacionadas as preferências de materiais e nível de conhecimento foram coletadas e utilizadas para gerar o estudo. A análise utilizou regra de associação com o algoritmo Apriori.

Em [Manhães et al. 2011] o autor apresenta algumas técnicas de mineração de dados com objetivo de classificar alunos propensos à evasão na Universidade Federal do Rio de Janeiro (UFRJ). A proposta é oferecer uma ferramenta para auxiliar os docentes na categorização do perfil de seus alunos, e possibilitar a realização de ações com o objetivo de evitar tal evasão. Contudo, o estudo considerou apenas variáveis relacionadas ao desempenho acadêmico, deixando de lado informações concernentes a instituição de ensino e questões sociais dos discentes. A pesquisa também propôs alguns algoritmos de classificação, a fim de identificar qual se adequaria melhor ao problema, e teve como resultado precisões variando entre $75 \%$ a $80 \%$. Vale ressaltar que entres os algoritmos utilizados, o que obteve melhor resultado nos experimentos foi o OneR.

\section{Metodologia}

\subsection{CRoss-Industry Standard Process for Data Mining (CRISP-DM)}

CRISP-DM é um modelo documentado e disponível livremente, onde são apresentadas boas práticas para se extrair resultados melhores no processo de mineração de dados [Shearer 2000]. O modelo aborda questões como a organização que a estrutura analisada precisa para gerar resultados melhores. Além de descrever detalhadamente todos os passos necessários para a construção de um projeto de mineração de dados.

Todo o processo é realizado em seis passos e cada um deles tem um papel bem definido para a finalização correta do planejamento. A primeira fase é a compreensão do negócio, esse é um passo importante, pois nele serão definidos os objetivos do projeto. 
A segunda fase é a concepção dos dados, onde é feita a coleta, descrição, exploração e verificação da qualidade dos dados. A terceira etapa é a preparação dos dados, onde são realizados os processos de limpeza, formatação e definição do conjunto de dados utilizado. No quarto estágio são definidas as técnicas para a construção do modelo, teste do modelo e por fim a construção do mesmo. No quinto passo são realizadas revisões sobre os resultados gerados e tomadas decisões para os próximos passos. Na última etapa é implementado todo o desenvolvimento do projeto em ambiente de produção.

\subsection{Base de Dados}

O INEP publica anualmente os dados do Censo Escolar em forma de dados abertos que podem ser livremente utilizados e redistribuídos, estando sujeito a citação de sua fonte e divulgados sob a mesma licença [Molloy 2011]. Os dados são divulgados a partir do ano de 1995 e disponibilizados em forma de tabelas no formato CSV (Comma-separated values). A partir desta divulgação foram obtidos os conjuntos de dados dos anos de 2014, 2015 e 2016 dos estados do Ceará e Sergipe utilizados deste estudo. O critério de escolha dos estados deu-se por meio de uma análise sobre os dados para identificar quem possuía a maior e menor taxa no ranking do Índice de Desenvolvimento da Educação Básica para os estados da região Nordeste [INEP 2017].

Através da análise foi possível identificar que o estado de Sergipe detinha o menor IDEB e o estado do Ceará possuía o maior em relação aos demais estados analisados. Os dados obtidos abrangem as escolas, turmas, os docentes e alunos de cada estado, e são disponíveis em um arquivo único de cada região do Brasil. A identificação dos estados analisados foi feita por meio de variáveis que representam a sigla e o código de cada estado, os quais são posteriormente usadas na etapa de pré-processamento. As variáveis da base de dados são listadas em um dicionário de dados do censo escolar que também descreve cada variável, seu tipo e como funciona o processo de importação no software utilizado no passo de separação dos dados.

\subsection{Pré-Processamento}

A etapa de pré-processamento foi dividida em três partes que consistem primeiramente na separação dos estados utilizados na análise dos demais contidos no arquivo da região. Em seguida realizou-se a modelagem do banco para realizar as consultas das variáveis consideradas pertinentes neste estudo. Após a modelagem, os dados separados foram exportados em um banco de dados. Por fim, veio a etapa de construção das consultas para exportação dos valores somado ao tratamento utilizando a ferramenta estatística $R$.

O processo de separação dos dados se deu utilizando o SPSS, um software para análise de dados da IBM [IBM 2016]. Inicialmente foram exportados os arquivos referente aos alunos, docentes, escolas e turmas da região nordeste no software de análise; em seguida definiu-se a condição para a separação, ou seja, foi escolhida a variável referente a sigla ou o código da região, e a ela se atribuiu o valor referente as regiões do Ceará e de Sergipe que a variável deveria possuir para que fosse separada. Ao final do processo, os resultado foram exportados no formato CSV para serem trabalhados na etapa seguinte.

O passo seguinte, consistiu na modelagem e criação de um banco de dados que fosse capaz de armazenar os valores de saída advindos do processo de separação dos dados. Neste passo foi conduzida uma modelagem que atendesse ao armazenamento de 
todas as variáveis. Para que não houvesse problema na inserção, os valores adicionados ao banco foram todos tratados como tipo texto, incluindo os valores do tipo inteiro.

O sistema gerenciador de banco de dados escolhido foi o PostgreSQL, utilizado para construir um banco contendo as tabelas de escolas, docentes, turmas e alunos de cada ano analisado. Após a construção, foi feita a importação dos arquivos do processo anterior e, com o auxílio do dicionário de dados do censo, conduziu-se uma análise sobre a base de dados dos três anos para selecionar as variáveis mais pertinentes ao estudo. A seleção somou um total de 61 variáveis que foram adicionadas a consulta do banco de dados. Ao total, três consultas foram criadas unindo as quatro entidades analisadas em apenas um resultado, que foi exportado em um arquivo CSV para cada ano estudado.

O último passo do pré-processamento foi o tratamento dos valores exportados do banco. Para isto foi utilizada a ferramenta estatística $\mathrm{R}$, onde os resultados foram analisados em pares. O primeiro par analisado foram os anos de 2014 e 2015 sobre os quais foram executadas uma sequência de funções para identificar se o aluno evadiu ou não no ano seguinte, ou seja, o tratamento consistia em uma verificação se aluno havia evadido no ano de 2015 considerando o conjunto de alunos do ano anterior. O processo se repetiu utilizando os dados de 2015 e 2016, e o resultado foi explicitado em uma coluna com o valor 1 para o aluno evadido e 0 para o aluno que não evadiu.

O tratamento identificou um percentual de evasão de $17 \%$ entre os anos de 2014 e 2015 e entre os anos de 2015 e 2016 . E ao final do tratamento foram realizados testes na mesma ferramenta para verificação da consistência dos resultados, em que não foi identificado nenhum erro referente aos alunos evadidos. Após o teste dos valores, o resultado do tratamento foi exportado em dois arquivos contendo as evasões dos anos de 2015 e 2016 para serem utilizados no processo de mineração de dados.

\subsection{Mineração de Dados}

A mineração de dados é um processo de análise projetado para exploração de dados de grande volume. Por meio de algoritmos, a mineração de dados busca descobrir padrões de comportamento, prever resultados e criar novas informações que podem responder perguntas que não podem ser respondidas por meio de técnicas simples de consulta e geração de relatórios [Han and Kamber 2006].

\subsubsection{Regressão Logística}

A Regressão Logística é um modelo genérico estatístico que procura descrever o relacionamento entre um variável dependente (saída ou resposta) com um conjuntos de variáveis independentes (predição) contínuas e/ou binárias que também são chamadas de covariáveis [Hosmer and Lemeshow 2000].

Essa regressão é perfeitamente aplicável para este estudo, pois a variável resposta/saída é uma variável binária representada com 1 para o aluno evadido e 0 para o aluno que não evadiu.

$$
\ln \left(\frac{p}{1-p}\right)=\beta_{0}+\beta_{1} X_{1}+\beta_{2} X_{2}+\ldots+\beta_{k} X_{k}
$$


VI Congresso Brasileiro de Informática na Educação (CBIE 2017)

Anais do XXVIII Simpósio Brasileiro de Informática na Educação (SBIE 2017)

No modelo apresentado na equação 1, $p$ é a probabilidade de ocorrência do evento, $X$ indica as variáveis independentes e $\beta$ são os coeficientes estimados. Os coeficientes indicam a importância de cada variável independente para a ocorrência do evento. Na equação 2 é apresentada a probabilidade de ocorrência para $\mathrm{P}$ dado um $x$ qualquer.

$$
P(x)=\frac{1}{1+e^{-\left(\alpha+\sum \beta i X i\right)}}
$$

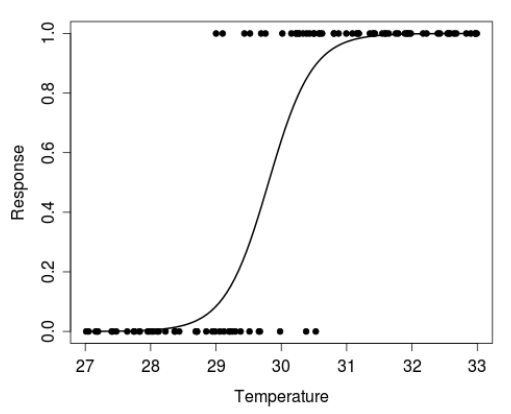

Figura 1. Cenário com relação confusa

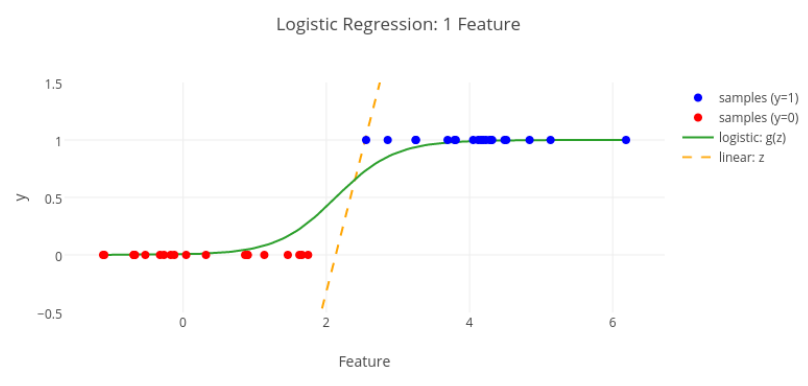

Figura 2. Cenário com relação bem definida

Na figura 1 é apresentado um cenário que não favorece a técnica de regressão, pois existem muitos valores que estão se sobrepondo. A figura 2 ilustra o cenário contrário, nela é exibido um cenário ideal, onde os dados de cada classe estão bem definidos.

A técnica de regressão foi executada no SPSS, que dispõe de funcionalidades para auxiliar a operação. A variável de evasão que foi obtida no último passo do préprocessamento, foi definida como dependente, e todos os outros elementos utilizados no estudo foram definidas como covariáveis.

\subsubsection{Indução de Regra}

A indução de regra é uma técnica de mineração de dados que fornece regras de um conjunto de dados, tendo o objetivo de encontrar padrões que são posteriormente expressos em regras se-então. A técnica extrai valores que representam o comportamento do conjunto de dados analisado [Maimon and Rokach 2010], e que é apresentado ao utilizador de acordo com a sua frequência. 
VI Congresso Brasileiro de Informática na Educação (CBIE 2017)

Anais do XXVIII Simpósio Brasileiro de Informática na Educação (SBIE 2017)

Normalmente as regras consistem em duas partes, a parte antecedente e a parte consequente. Elas são expressas na forma condicional lógica "se (n condições) então (decisão)", e uma vez extraídas do conjunto de dados sua utilização vai variar conforme seu objetivo. Elas podem ser usadas com o objetivo no antecedente, ou seja, as regras coletadas serão as que possuam valor para o antecedente. Outro exemplo é objetivando o consequente, onde as regras que possuem algum valor para o consequente são selecionadas para ajudar no entendimento do que está associado e do que afeta o consequente [Gilmar 2000]. Na análise, o foco será o consequente, visando encontrar cenários com altas taxas de evasão, para que sejam adotadas medidas contra eles, e as regras associadas com baixas taxas, para que sejam identificadas as características que representam esta condição.

O estudo fez uso do algoritmo RIPPER (Repeated Incremental Pruning to Produce Error Reduction) ou Poda Incremental Repetida para Produzir Redução de Erro. A sua aplicação foi feita por meio do software RapidMiner, o qual é ferramenta para mineração de dados de código aberto. O algoritmo utilizado teve como entrada os dados advindos da fase de pré-processamento e teve como saída as regras representando o padrão de comportamento do conjunto de dados, a taxa total de acerto das regras sobre todo o conjunto de dados e o acerto de cada regra.

\section{Resultados}

\subsection{Regressão Logística}

O modelo de regressão logística mostrou-se eficiente para a analisar os dados dos dois estados, visto que há uma grande diferença entre o número de alunos e rendimento no IDEB, a acurácia de classificação resultou em média 87.4 no Ceará e 86.8 em Sergipe.

Em geral as variáveis relacionadas às etapas de ensino mostraram-se significantes de forma positiva ou negativa a evasão escolar. As etapas do ensino médio apresentaramse de forma positiva, ou seja, há maior probabilidade de evasão. Com acesso completo ao relatório é possível inferir quais etapas precisam de maior foco em ações para prevenção da evasão.

Para os estado do Ceará em todos os anos, a raça negra foi uma única variável que influenciou positivamente, contudo em Sergipe esse fator não gerou significância alguma para a variável dependente. Nos dois estados, a variável idade gerou influência positiva, o que significa dizer que a cada ano a probabilidade de desistência torna-se maior para o aluno. Essa probabilidade muda entre os anos e os estados, no entanto apresentam-se com uma diferença mínima.

No estado do Ceará no par 2014-2015 a variável que informa se a escola possui laboratório de informática influenciou de forma positiva, contudo no ano seguinte o valor já apresentou-se invertido, mostrando que houve algum investimento em laboratórios para essa reversão. Nas duas bases com o estado de Sergipe a influência apresentou-se de negativa.

A tabela 1 apresenta algumas variáveis e seus coeficientes que mostraram-se importantes. Os coeficientes que não apresentaram significância foram descartados.

\subsection{Regra de Indução}

O processo de análise utilizando a indução de regra obteve como resultado um total de 284 regras geradas para todo o conjunto de dados utilizado nesta pesquisa. A partir dos resul- 
VI Congresso Brasileiro de Informática na Educação (CBIE 2017)

Anais do XXVIII Simpósio Brasileiro de Informática na Educação (SBIE 2017)

\begin{tabular}{|l|c|c|c|c|}
\hline \multicolumn{1}{|c|}{ Variáveis } & \multicolumn{2}{c|}{ Ceará } & \multicolumn{2}{c|}{ Sergipe } \\
\hline \multicolumn{1}{|c|}{} & $\mathrm{B}(2014 / 2015$ & $\mathrm{B}(2015 / 2016)$ & $\mathrm{B}(2014 / 2015)$ & $\mathrm{B}(2015 / 2016)$ \\
\hline aluno possui necessidade especial & 0,259 & 0,217 & 0,018 & $-0,092$ \\
\hline aluno reside na zona urbana & $-0,05$ & $-0,102$ & 0,001 & 0,29 \\
\hline docentes mestres & $-0,589$ & $-0,249$ & - & $-0,184$ \\
\hline docentes com contrato temporário & 0,522 & 0,460 & - & - \\
\hline docentes com nível fundamental incompleto & 0,620 & 0,438 & - & 0,0436 \\
\hline
\end{tabular}

Tabela 1. Regressão Logística

tados, foi aplicado um filtro para seleção das regras que apareciam com mais frequência. O critério utilizado foi a seleção das regras com mais de mil ocorrências tanto para alunos evadidos ou para alunos que não evadiram. Dessa forma, foi possível identificar as principais variáveis relacionadas à evasão escolar descobertas por este método.

De maneira geral, a idade do aluno, a etapa de ensino na qual ele se encontra e as variáveis que caracterizam a existência ou não de laboratórios, auditório ou até transporte público foram as variáveis que apareceram com maior frequência na análise. Somado a elas, mais variáveis foram encontradas, no entanto, elas não aparecem com tanta frequência ou apresentam uma evasão elevada como as citadas anteriormente.

A tabela 2 contém as principais regras concernentes aos pares de anos 2014-2015, 2015-2016 de Sergipe, e a tabela 3 apresenta as regras do Ceará para os mesmos pares de anos.

\begin{tabular}{|c|c|c|c|}
\hline Ano & Condições da Regra & Quantidade evadida & Quantidade não evadida \\
\hline \multirow{3}{*}{2014} & Idade $\leq 15.500$ & 36.491 & 418.895 \\
\cline { 2 - 4 } e & Idade $\leq 17.500$ E Etapa de Ensino = 2 ${ }^{\circ}$ Ano Médio & 861 & 12.655 \\
\cline { 2 - 4 } 2015 & Idade $>20.500$ E Modalidade de Ensino = EJA E Idade $\leq 28.500$ & 5.972 & 3.113 \\
\cline { 2 - 4 } 2015 & Idade $>20.500$ E Ensino Médio Profissionalizante $=$ SIM & 7.039 & 3004 \\
\cline { 2 - 4 } e & Idade $\leq 14.500$ & 33.332 & 7.932 \\
\cline { 2 - 4 } 2016 & Sala de Atendimento Especial = NÂO E Laboratório de Ciências = NÃO & 57.196 & 313.371 \\
\cline { 2 - 4 } & Localização da Escola = Rural E Idade $>20$ & 2.886 & 1.467 \\
\hline
\end{tabular}

Tabela 2. Regras do Estado de Sergipe

Os dados de Sergipe mostraram que o índice de evasão para alunos com idade menor ou igual a 14 e 15 anos foi bem baixa nos dois pares de anos analisados, chegando a representar um percentual de mais de $60 \%$ dos alunos não evadidos considerando apenas estas regras. Outro ponto importante foram as etapas de ensino que aparecem em duas regras mostrando que o índice de evasão no primeiro e no segundo ano do ensino médio são baixos. Os fatores que apresentaram o índice de evasão alto foram escolas sem sala de atendimento especial que atendem a alunos com necessidades especiais, é a ausência de laboratório de ciências na instituição. A idade do aluno relacionada com a modalidade de ensino ou se o aluno estuda em uma instituição com ensino médio profissionalizante também foi fator influente na evasão.

Os dados do estado do Ceará apresentaram algumas diferenças em relação aos de Sergipe. As regras contendo a condições onde o estudante possui idade menor ou igual a 18 ou 19 anos e meio foram as que apresentaram os menores índices de evasão. A primeira regra da tabela 3 exemplifica este índice, onde é mostrado que escolas com dependência administrativa municipal e com alunos de idade menor ou igual a 18 anos e meio possuem taxas de evasão muito baixas, chegando a representar um percentual de $56 \%$ de alunos não evadidos quando esta regra é aplicada em todo o conjunto de dados. 
VI Congresso Brasileiro de Informática na Educação (CBIE 2017)

Anais do XXVIII Simpósio Brasileiro de Informática na Educação (SBIE 2017)

\begin{tabular}{|c|c|c|c|}
\hline Ano & Condições da Regra & Quantidade evadida & Quantidade não evadida \\
\hline \multirow{3}{*}{2014} & Dependência da Escola $=$ Municipal E Idade $\leq 18.500$ & 141.350 & 1.519 .411 \\
\hline & Etapa de Ensino $=2^{\circ}$ Ano Médio E Idade $\leq 19.500$ & 13.377 & 78.371 \\
\hline & $\begin{array}{c}\text { Modalidade de Ensino }=\text { EJA E } \\
\text { Número de Computadores na Escola } \leq 33.500\end{array}$ & 71.877 & 48.571 \\
\hline $\begin{array}{c}\mathrm{e} \\
2015\end{array}$ & $\begin{array}{c}\text { Idade }>20.500 \text { E Número de Salas na Escola } \leq 26.500 \text { E } \\
\text { Escola Com Ensino Médio Profissionalizante }=\text { SIM }\end{array}$ & 10.743 & 2.808 \\
\hline \multirow{4}{*}{$\begin{array}{c}\mathrm{e} \\
2016\end{array}$} & Idade $\leq 15.500$ & 166.253 & 1.739 .567 \\
\hline & $\begin{array}{c}\text { Idade } \leq 19.500 \text { E Laboratório de Ciências }=\text { SIM E } \\
\text { Etapa de Ensino }=2^{\circ} \text { Ano Médio }\end{array}$ & 1.296 & 3.129 \\
\hline & Possui Creche $=$ NAO E Número de Alunos na Turma $>24.500$ & 7.071 & 5.740 \\
\hline & Sala de Atendimento Especial $=$ NÃO E Laboratório de Ciências $=$ NÃO & 196.689 & 1.125 .438 \\
\hline
\end{tabular}

Tabela 3. Regras do Estado do Ceará

Condições como a ausência de laboratório de ciências, sala de atendimento foram variáveis também encontradas no conjunto de dados do Ceará. Além disso, a análise também apresentou variáveis que não aparecem nos resultados de Sergipe, mas que também se relacionam com a evasão, como a quantidade de alunos por turma, a existência ou não de creche na escola e o número de computadores na instituição.

\section{Considerações finais}

Este trabalho apresentou um estudo sobre as variáveis que possuem maior influência na evasão escolar tendo como base os dados fornecidos nos Censos Escolares dos anos 2014, 2015 e 2016 para os estados Ceará e Sergipe. A metodologia CRISP-DM foi utilizada para compreensão, preparação dos dados, definição de técnicas e análise de resultados. Regressão Logística e Indução de Regra foram as técnicas de mineração de dados utilizadas neste trabalho para identificar as variáveis que mais influenciam a evasão escolar. Variáveis como idade, etapa de ensino, modalidade de ensino, existência de laboratórios e localização da escola se destacaram na criação das regras que influenciam na evasão escolar.

Outros estados podem ser considerados em trabalhos futuros para verificar se os resultados encontrados ocorrem em toda a região Nordeste, por exemplo. Além disso, outras técnicas de mineração de dados podem ser utilizadas para prever a evasão escolar. A evasão escolar é provocada por inúmeros fatores, portanto, estudos para compreender melhor essas causas são fundamentais e podem contribuir na elaboração de políticas públicas para reduzir esse problema.

\section{Referências}

Agaoglu, M.(2016). Predicting instructor performance using data mining techniques in higher education. IEEE Access, 4:2379-2387.

Bezerra, C., Scholz, R., Adeodato, P., Pontes, T., and Silva, I.(2016). Evasão escolar: Aplicando mineração de dados para identificar variáveis relevantes. In Anais do XXVII Simpósio Brasileiro de Informática na Educação (SBIE 2016).

Brasil(2006). Alunas e alunos da eja. http://portal.mec.gov.br/secad/arquivos/pdf/eja caderno1.pdf. Acessado: 14/05/2017.

BRASIL(2012). Índice de abandono escolar é três vezes maior no $6^{\circ}$ ano do ensino fundamental. 
VI Congresso Brasileiro de Informática na Educação (CBIE 2017)

Anais do XXVIII Simpósio Brasileiro de Informática na Educação (SBIE 2017)

Digiampietri, L. A., Nakano, F., and Lauretto, M. S.(2016). Mineração de dados para identificação de alunos com alto risco de evasão: Um estudo de caso. Grad+ - Revista de Graduação da USP, 1(1).

Diniz, G. M., Machado, D. d. Q., and Moura, H. J.(2014). Políticas públicas de combate ao analfabetismo no brasil: uma investigação sobre a atuação do programa brasil alfabetizado em municípios do ceará. Revista de Administração Pública, 48:641 - 666.

Gilmar, W. S. A.(2000). Data mining aplicado ao serviço público, extração de conhecimento das ações do ministério público brasileiro.

Han, J. and Kamber, M.(2006). Data Mining: Concepts and Techniques. Morgan Kaufmann Publishers Inc., San Francisco, CA, USA, 2nd edition.

Hosmer, D. W. and Lemeshow, S.(2000). Applied logistic regression. "A WileyInterscience publication.”.

IBM(2016). Ibm spss statistics 24 brief guide. Disponível em: http: //public.dhe.ibm.com/software/analytics/spss/documentation/statistics/24.0/pt-BR/ client/Manuals/IBM_SPSS_Statistics_Brief_Guide.pdf. Acessado em: 07/04/2017.

INEP(2017). Ideb - resultados e metas. Disponível em: http://ideb.inep.gov.br/resultado/home.seam?cid=1005260. Acessado em: 07/04/2017.

Maimon, O. and Rokach, L.(2010). Data Mining and Knowledge Discovery Handbook. Series in Solid-State Sciences. Springer US.

Manhães, L. M. B., Cruz, S. M. S., Costa, R. J. M., and Zimbrão, J. Z. G.(2011). Previsão de estudantes com risco de evasão utilizando técnicas de mineração de dados.

Molloy, J. C.(2011). The open knowledge foundation: Open data means better science. Plos Biology, 9(12).

Nunes, Felipe Becker; Voss, G. C. S.(2015). Mineração de dados educacionais e mundos virtuais: um estudo explorativo no opensim.

OECD(2015). Programme for international student assment (pisa) results from pisa 2015.

Shearer, C.(2000). The crisp-dm model: The new blueprint for data mining. Journal of Data Warehousing, 5(4). 